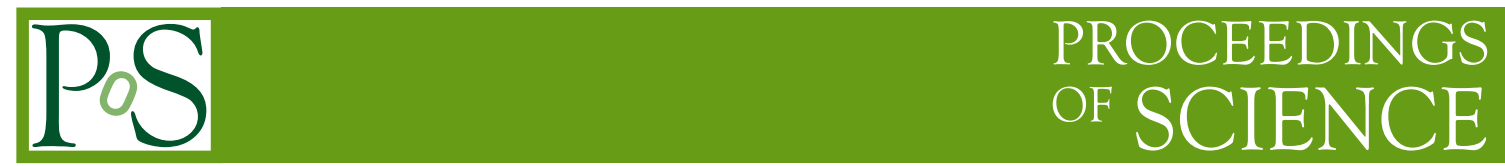

\title{
Vacuum magnetic birefringence
}

\author{
Agathe Cadène, Paul Berceau, Mathilde Fouché, Remy Battesti, Carlo Rizzo* \\ Laboratoire National des Champs Magnétiques Intenses (UPR 3228, \\ CNRS-UPS-UJF-INSA), F-31400 Toulouse Cedex, France, EU
}

E-mail: [arle.rizzodnemi.cnrs.fr

\begin{abstract}
We present the measurement of the vacuum magnetic linear birefringence obtained using the first generation setup of the BMV experiment. Our apparatus is based on an up-to-date resonant optical cavity coupled to a transverse magnetic field. The reported value of vacuum magnetic linear birefringence $k_{\mathrm{CM}}$, obtained with about 100 magnetic pulses and a maximum field of $6.5 \mathrm{~T}$, is $k_{\mathrm{CM}}=(5.1 \pm 6.2) \times 10^{-21} \mathrm{~T}^{-2}$ at $3 \sigma$ confidence level. This result is also used to extend the excluded region of axion-two photons coupling constant $g$ as a function of the axion mass $m_{\mathrm{a}}$. The best limit is obtained at $m_{\mathrm{a}}=3.3 \mathrm{meV}$ with $g<1.9 \times 10^{-6} \mathrm{GeV}^{-1}$ at $3 \sigma$ confidence level.
\end{abstract}

Photon 2013,

20-24 May 2013

Paris, France

* Speaker. 


\section{Introduction}

In the presence of a transverse external magnetic field $\boldsymbol{B}$, any medium shows a linear birefringence. The existence of such a magnetic linear birefringence is also predicted in vacuum through the Quantum ElectroDynamics (QED) Heisenberg-Euler effective lagrangian (see Ref. [W] and references therein). In a vacuum therefore the index of refraction $n_{\|}$for light polarized parallel to $\boldsymbol{B}$ is expected to be different from the index of refraction $n_{\perp}$ for light polarized perpendicular to $\boldsymbol{B}$ such that [四:

$$
\Delta n_{\mathrm{CM}}=n_{\|}-n_{\perp}=k_{\mathrm{CM}} B^{2} .
$$

The experimental proof of such a very fundamental QED prediction is still lacking [U]. At the first order in the fine structure constant $\alpha, k_{\mathrm{CM}}$ can be written as:

$$
k_{\mathrm{CM}}=2 \alpha^{2} \hbar^{3} / 15 \mu_{0} m_{\mathrm{e}}^{4} c^{5}
$$

with $\hbar$ the Planck constant over $2 \pi, m_{\mathrm{e}}$ the electron mass, $c$ the speed of light in vacuum, and $\mu_{0}$ the magnetic constant. Using the CODATA recommended values for fundamental constants [ [ $]$ ], one obtains $k_{\mathrm{CM}} \sim 4.0 \times 10^{-24} \mathrm{~T}^{-2}$.

In our experiment, we measure $\Delta n_{\mathrm{CM}}$ via the ellipticity $\psi$ induced on a linearly polarized light propagating in the birefringent vacuum:

$$
\psi=\pi k_{\mathrm{CM}} \frac{L_{B}}{\lambda} B^{2} \sin 2 \theta_{\mathrm{P}},
$$

where $\lambda$ is the light wavelength, $L_{B}$ is the path length in the magnetic field, and $\theta_{\mathrm{P}}=45^{\circ}$ is the angle between the light polarization and the birefringence axis. This equation clearly shows that the critical experimental parameter is the product $B^{2} L_{B}$. In order to increase the ellipticity to be measured, we use an optical cavity to store light in the magnetic field region as long as possible. The total acquired ellipticity $\Psi$ is linked to the ellipticity $\psi$ acquired in the absence of cavity and depends on the cavity finesse $F$ as $\Psi=\frac{2 F}{\pi} \psi$.

In this contribution to Photon 2013, we present a measurement of $k_{\mathrm{CM}}$ obtained using the first generation setup of the BMV (Biréfringence Magnétique du Vide) experiment at the National High Magnetic Field Laboratory of Toulouse, France (LNCMI-T). The reported value of $k_{\mathrm{CM}}$ was obtained with 101 magnetic pulses and a maximum field of $6.5 \mathrm{~T}$. This result corresponds to one of the most precise measurement ever realized. It is therefore a clear validation of our innovative experimental method. Finally, this kind of experiment can also give limits on axion-like particles coupled to two photons travelling through a transverse magnetic field. The value of the vacuum magnetic birefringence is used to extend the excluded region of axion-two photons coupling constant $g$ as a function of the axion mass $m_{\mathrm{a}}$.

\section{Experimental setup}

Our experimental setup is described in Refs. [B], 田, 目]. A linearly polarized Nd:YAG laser beam $(\lambda=1064 \mathrm{~nm})$ goes through an acousto-optic modulator (AOM) used in double passage for an adjustment of the laser frequency. It is then injected into a monomode optical fiber before entering a high finesse Fabry-Pérot cavity of length $L_{\mathrm{c}}=2.27 \mathrm{~m}$, consisting of the mirrors $\mathrm{M}_{1}$ and 


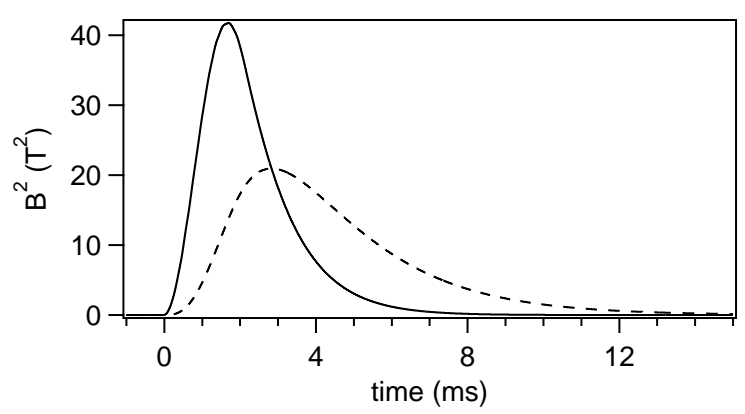

Figure 1: Square of the magnetic field amplitude as a function of time for a maximum field of $6.5 \mathrm{~T}$. Solid black curve, $B^{2}$; dashed curve, $B_{\mathrm{f}}^{2}$.

$\mathrm{M}_{2}$. This corresponds to a cavity free spectral range of $\Delta^{\mathrm{FSR}}=c / 2 L_{\mathrm{c}}=65.996 \mathrm{MHz}$. The laser passes through an electro-optic modulator (EOM) creating sidebands at $10 \mathrm{MHz}$. We analyze the beam reflected by the cavity on the photodiode $\mathrm{Ph}_{\mathrm{r}}$. This signal is used to lock the laser frequency to the cavity resonance frequency using the Pound-Drever-Hall method [6]. All the optical devices from the polarizer to the analyzer are placed in an ultrahigh-vacuum chamber. During operation, the pressure inside the UHV vessel was about $10^{-7}$ mbar.

To measure the ellipticity induced by the Cotton-Mouton effect one needs a transverse magnetic field as high as possible. This is fulfilled using pulsed fields delivered by one magnet, named $\mathrm{X}$-coil, especially designed in our laboratory. The principle of this magnet and its properties are described in details in Refs. [廿, 团]. Data have been taken with a maximum magnetic field of 6.5 T, over an equivalent length $L_{B}$ of $0.137 \mathrm{~m}$ [B] , reached within $1.70 \mathrm{~ms}$ while the total duration of a pulse is less than $10 \mathrm{~ms}$ as shown in Fig. W. Moreover, we can remotely switch the high-voltage connections to reverse $\boldsymbol{B}$ in order to set it parallel or antiparallel to the $x$ direction. The maximum repetition rate is 6 pulses per hour.

We infer the cavity finesse from the measurement of the photon lifetime $\tau$ [B]]. We get $\tau=$ $1.07 \mathrm{~ms}$ and consequentely $F=445000$. This corresponds to a cavity linewidth $\Delta v=c / 2 F L_{\mathrm{c}}$ of $148 \mathrm{~Hz}$. This is one of the sharpest infrared cavity in the world [3]].

Before entering the Fabry-Pérot cavity, light is polarized by the polarizer P. The beam transmitted by the cavity is then analyzed by the analyzer A crossed at maximum extinction. We extract both polarizations: parallel and perpendicular to $\mathrm{P}$. The extraordinary ray, whose polarization is perpendicular to the incident polarization, is detected by the photodiode $\mathrm{Ph}_{\mathrm{e}}\left(\right.$ power $I_{\mathrm{e}}$ ), while the ordinary ray, whose polarization is parallel to the incident polarization, is detected by $\mathrm{Ph}_{\mathrm{t}}$ (power $\left.I_{\mathrm{t}}\right)$. The ellipticity $\Psi(t)$ induced by the transverse magnetic field is related to the ratio of the extraordinary and ordinary powers as follows:

$$
\frac{I_{\mathrm{e}}(t)}{I_{\mathrm{t}, \mathrm{f}}(t)}=\sigma^{2}+[\Gamma+\Psi(t)]^{2} \simeq \sigma^{2}+\Gamma^{2}+2 \Gamma \Psi(t) \text { for } \Psi \ll \Gamma,
$$

with $\sigma^{2}$ the polarizer extinction ratio and $\Gamma$ the total static ellipticity. This static ellipticity is due to the mirrors' intrinsic phase retardation [[]]. 
To measure the polarizer extinction ratio, we first set $\Gamma=0$, with no magnetic field. We get $I_{\mathrm{e}} / I_{\mathrm{t}, \mathrm{f}}=\sigma^{2} \sim 7 \times 10^{-7}$. Starting from $\Gamma=0$ and rotating $\mathrm{M}_{1}$ in the clockwise or counterclockwise direction, we choose the value of $\Gamma$ as well as its sign determined by $\mathrm{CM}$ measurements in nitrogen and helium gas. The measurement of $\sigma^{2}$ and the adjustment of the value and sign of $\Gamma$ are done before each magnetic pulse.

Due to the photon lifetime, the cavity acts as a first order low pass filter, as explained in details in Ref. [9]. The ellipticity $\Psi$ induced by the external magnetic field is thus $\Psi(t)=\alpha B_{\mathrm{f}}^{2}(t)$, where the filtered field $B_{\mathrm{f}}^{2}$ is calculated from $B^{2}$ taking into account the cavity filtering. The time profile of $B_{\mathrm{f}}^{2}$ is plotted in Fig. W with the dashed curve. In particular, we see that the cavity filtering induces an attenuation and a shift of the maximum. The cavity filtering has also to be applied to $I_{\mathrm{t}}$ as explained in details in Refs. [9, [5].

The calculated signals used for the analysis are described in details in Ref. [[]]. In order to extract the ellipticity $\Psi(t)$ from Eq. (미), we calculate the following $Y(t)$ signal after each pulse:

$$
Y(t)=\frac{\frac{I_{\mathrm{e}}(t)}{I_{\mathrm{t}, \mathrm{f}}(t)}-I_{\mathrm{dc}}}{2|\Gamma|} \simeq \gamma \Psi(t),
$$

where $\gamma$ corresponds to the sign of $\Gamma$. The absolute value of the cavity ellipticity is measured a few milliseconds before each magnetic pulse thanks to the following equation:

$$
|\Gamma|=\sqrt{\left.\left\langle\frac{I_{\mathrm{e}}(t)}{I_{\mathrm{t}, \mathrm{f}}(t)}\right\rangle\right|_{t_{\Gamma}<t<0}-\sigma^{2} .}
$$

where $t_{\Gamma}$ corresponds to the beginning of the analysis and $t=0$ to the beginning of the applied magnetic field.

Signals $Y(t)$ are acquired for both signs of $\Gamma$ and for both directions of $\boldsymbol{B}$ : parallel to $x$ is denoted as $>0$ and antiparallel is denoted as $<0$. This gives four data series: $(\Gamma>0, B>0)$, $(\Gamma>0, B<0),(\Gamma<0, B<0)$ and $(\Gamma<0, B>0)$. For each series, signals are averaged and denoted as $Y_{>>}, Y_{><}, Y_{<<}$and $Y_{<>}$. The first subscript corresponds to $\Gamma>0$ or $<0$ while the second one corresponds to $\boldsymbol{B}$ parallel or antiparallel to $x$.

\section{Data analysis and results}

A few milliseconds after the beginning of the pulse a perturbation due to the sound propagating from the coil to the cavity mirrors induces an ellipticity noise which degrades our sensitivity. To avoid this we stop the analysis at $t=3.1 \mathrm{~ms}$. Symmetrically, we start the analysis at $t_{\Gamma}=-3.1 \mathrm{~ms}$.

From the 101 pulses, we calculate the signals $Y_{>>}, Y_{><}, Y_{<<}$, and $Y_{<>}$, denoted $Y_{\mathrm{j}}$ with $\mathrm{j}=>>$ ,$><,>>,<>$. They correspond to the average of the $Y(t)$ for each of the four series. The $Y_{\mathrm{j}}$ uncertainties are calculated at each time $t_{\mathrm{i}}$ as $\Delta Y_{\mathrm{j}}\left(t_{\mathrm{i}}\right)=\sigma_{\mathrm{j}}\left(t_{\mathrm{i}}\right) / \sqrt{N_{\mathrm{j}}}$, with $\sigma_{\mathrm{j}}\left(t_{\mathrm{i}}\right)$ the standard deviation of the $Y_{\mathrm{j}}\left(t_{\mathrm{i}}\right)$ distribution and $N_{\mathrm{j}}$ the number of shots for the $\mathrm{j}$ series.

If no systematic effects affect the experiment, we can fit the $Y_{\mathrm{j}}(t)$ averaged signals by $\gamma \alpha B_{\mathrm{f}}^{2}(t)$. The Cotton-Mouton constant $k_{\mathrm{CM}}$ is finally deduced from the measured experimental parameters as follows [B]]:

$$
k_{\mathrm{CM}}=\frac{\alpha}{4 \pi \tau \Delta^{\mathrm{FSR}}} \frac{\lambda}{L_{B}} \frac{1}{\sin 2 \theta_{\mathrm{P}}}
$$


Actually, as explained in details in Ref. [[]], one has to consider systematic effects that mimic the $\mathrm{CM}$ effect we want to measure. We thus derive a more general expression taking into account the symmetry properties of $Y_{\mathrm{j}}$ towards experimental parameters:

$$
\begin{aligned}
Y_{>>} & =a_{>>} S_{++}+b_{>>} S_{+-}+c_{>>} S_{--}+d_{>>} S_{-+}, \\
& =a_{>>} S_{++}+b_{>>} S_{+-}+c_{>>} S_{--}+\Psi, \\
Y_{><} & =a_{><} S_{++}-b_{><<} S_{+-}-c_{><<} S_{--}+d_{><} S_{-+}, \\
& =a_{><<} S_{++}-b_{><} S_{+-}-c_{><} S_{--}+\Psi, \\
Y_{<<} & =a_{<<} S_{++}-b_{<<} S_{+-}+c_{<<} S_{--}-d_{<<} S_{-+}, \\
& =a_{<<} S_{++}-b_{<<} S_{+-}+c_{<<} S_{--}-\Psi, \\
Y_{<>} & =a_{<>} S_{++}+b_{<>} S_{+-}-c_{<>} S_{--}-d_{<>} S_{-+}, \\
& =a_{<>} S_{++}+b_{<>} S_{+-}-c_{<>} S_{--}-\Psi .
\end{aligned}
$$

The $S$ functions correspond to a given symmetry towards the sign of $\Gamma$ and the direction of $\boldsymbol{B}$. The first subscript + (resp. - ) indicates an even (resp. odd) parity with respect to the sign of $\Gamma$. The same convention is used for the second subscript corresponding to $\boldsymbol{B}$. CM effect signal contributes to $S_{-+}$since it depends on the cavity birefringence $\Gamma$ and on the square of the magnetic field amplitude as shown in Eqs. (ㅍ.3) and (ㄹ.2). We can thus replace $d S_{-+}$by $\gamma \Psi$.

The $S$ functions can be extracted with a linear combination of $Y_{\mathrm{j}}$ as follows:

$$
\begin{aligned}
J_{1} & \equiv \frac{Y_{>>}+Y_{><}+Y_{<<}+Y_{<>}}{4}, \\
& =\bar{a} S_{++}+\Delta b_{1} S_{+-}+\Delta c_{1} S_{--}+\Delta d_{1} S_{-+}, \\
J_{2} & \equiv \frac{Y_{>>}-Y_{><-}-Y_{<<}+Y_{<>}}{4}, \\
& =\Delta a_{2} S_{++}+\bar{b} S_{+-}+\Delta c_{2} S_{--}+\Delta d_{2} S_{-+}, \\
J_{3} & \equiv \frac{Y_{>>}-Y_{><+}+Y_{<<}-Y_{<>}}{4}, \\
& =\Delta a_{3} S_{++}+\Delta b_{3} S_{+-}+\bar{c} S_{--}+\Delta d_{3} S_{-+}, \\
J_{4} & \equiv \frac{Y_{>>}+Y_{><-} Y_{<<-} Y_{<>}}{4}, \\
& =\Delta a_{4} S_{++}+\Delta b_{4} S_{+-}+\Delta c_{4} S_{--}+\bar{d} S_{-+} .
\end{aligned}
$$

$J_{1}(t), J_{2}(t), J_{3}(t)$ and $J_{4}(t)$ are plotted in Fig. $\square$. Their uncertainties are calculated from the $Y_{\mathrm{j}}$ uncertainties. The weighting parameters $a, b, c$ and $d$ depend on the experimental adjustment from pulse to pulse and from day to day. Their relative variations are small: $\Delta a / \bar{a}, \Delta b / \bar{b}, \Delta c / \bar{c}, \Delta d / \bar{d} \ll$ 1. $\Delta a, \Delta b$ and $\Delta c$ are mainly due to the $\Gamma$ variation from one shot to another.

We thus write:

$$
J_{1} \simeq \bar{a} S_{++}, J_{2} \simeq \bar{b} S_{+-}, J_{3} \simeq \bar{c} S_{--}, J_{4} \simeq \frac{\Delta a_{4}}{\bar{a}} J_{1}+\frac{\Delta b_{4}}{\bar{b}} J_{2}+\frac{\Delta c_{4}}{\bar{c}} J_{3}+\Psi .
$$

We then calculate:

$$
J_{4}^{\prime} \equiv J_{4}-\left[\frac{\Delta a_{4}}{\bar{a}} J_{1}+\frac{\Delta b_{4}}{\bar{b}} J_{2}+\frac{\Delta c_{4}}{\bar{c}} J_{3}\right] \simeq \Psi
$$



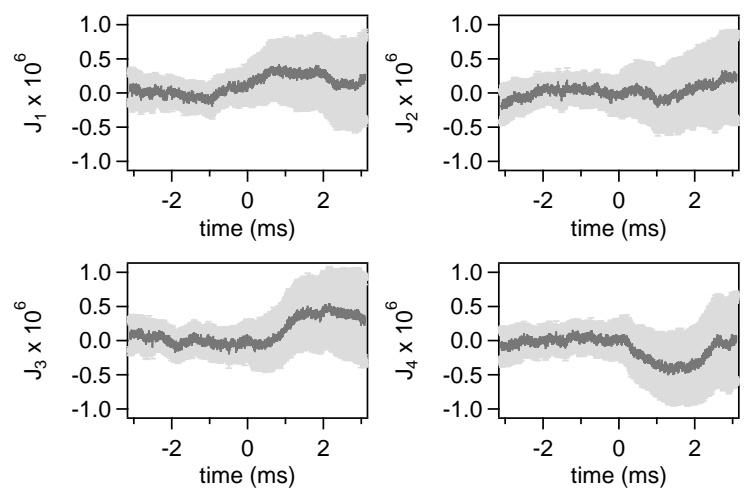

Figure 2: Time evolution of $J_{1}, J_{2}, J_{3}$ and $J_{4}$ (dark grey curve) and their uncertainties at $3 \sigma$ confidence level (light grey).

which corresponds to the Cotton-Mouton signal.

This method has been validated measuring Helium gas magnetic birefringence as reported in ref. [5]]. In fig. [] we show the $J_{4}$ signal obtained for $16210^{-3}$ atm of Helium gas.

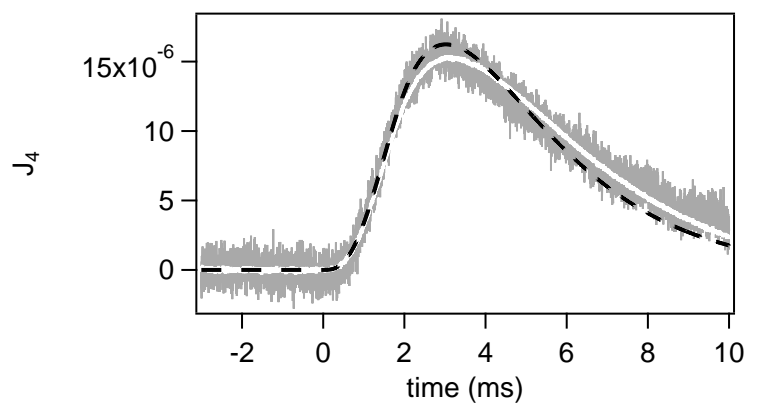

Figure 3: Gray: Time evolution of $J_{4}$ for a pressure of $162 \times 10^{-3} \mathrm{~atm}$. Black dashed curve: fit with $\alpha B_{\mathrm{f}}^{2}$. White solid curve: fit with $\alpha B_{\mathrm{f}}^{2}+$ corrections due to the relative variations of weighting parameters $a, b, c$ and $d$, the value of $\alpha$ being fixed at the value obtained with the previous fit $\alpha B_{\mathrm{f}}^{2}$.

Nevertheless, as we see in Fig. 凤, the major component of $J_{4}^{\prime}$ is not $\alpha B_{\mathrm{f}}^{2}$ but a supplementary systematic effect.

The setup is subject to several mechanical resonances which can be excited both by the environment and the magnetic field. The latter could thus trigger a mechanical oscillation of the setup at $t=0$. We try to fit $J_{4}^{\prime}$ by a sine function starting at $t=0$. The fit gives a frequency of $(180 \pm 3) \mathrm{Hz}$ and it is superimposed to $J_{4}^{\prime}$ in Fig. 因. We finally fit the residues by $\alpha B_{\mathrm{f}}^{2}$ and we obtain:

$$
k_{\mathrm{CM}}^{\text {noisefloor }}=(-0.9 \pm 6.2) \times 10^{-21} \mathrm{~T}^{-2},
$$

at $3 \sigma$ confidence level. This corresponds to our noise floor, which is half the one of the PVLAS collaboration in 2012 obtained with an integration time of $8192 \mathrm{~s}$ [ए]]].

In order to assess more precisely the physical origin of the systematic effect, we look to the power spectral density of $\Psi$. We find several resonances at $177 \mathrm{~Hz}, 200 \mathrm{~Hz}$ and above. The signal $J_{4}^{\prime}$ 


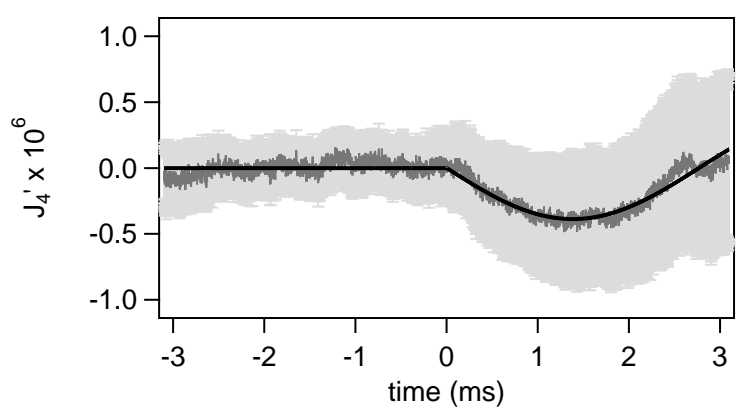

Figure 4: Time evolution of $J_{4}^{\prime}$ and its residues (dark grey). The $3 \sigma$ uncertainties are superimposed in light grey. Black curve: fit with a sine function at $180 \mathrm{~Hz}$.

is then fitted by a sine functions but with the frequency fixed to each of the resonance frequencies. The best fit, corresponding to the best $\chi^{2}$, is obtained for $177 \mathrm{~Hz}$, which is compatible with the frequency given by the previous fit. Fitting the residues by $\alpha B_{\mathrm{f}}^{2}$ gives our final value for the CM constant:

$$
k_{\mathrm{CM}}=(5.1 \pm 6.2) \times 10^{-21} \mathrm{~T}^{-2},
$$

at $3 \sigma$ confidence level.

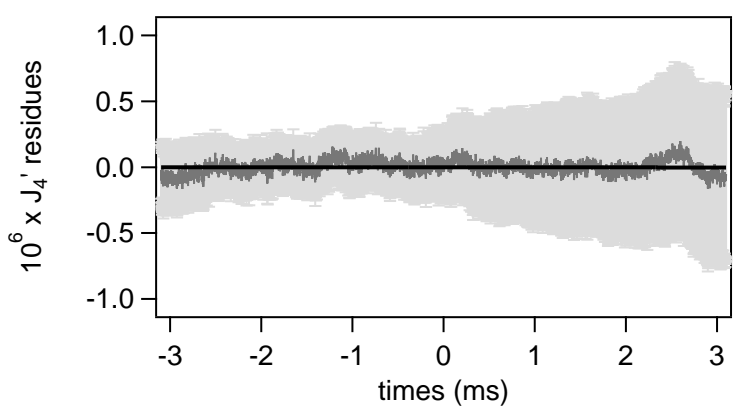

Figure 5: Time evolution of $J_{4}^{\prime}$ residues. The $3 \sigma$ uncertainties are superimposed in light grey. Black curve: fit with $\alpha B_{\mathrm{f}}^{2}$ function.

Our $k_{\mathrm{CM}}$ value is compatible with the expected one for the vacuum. We compare it to the other published values in Fig. 6. Our value is the most precise value ever realized.

Actually, after the theoretical calculations in the 70s, a first measurement of the $k_{\mathrm{CM}}$ value was published by the BFRT collaboration []. It It was based on a superconducting magnet providing a maximum field of $3.9 \mathrm{~T}$, and a multipass optical cavity. Spurious signals were always present (see Table $\mathrm{V}(\mathrm{b})$ in [प]]). Final results gave $k_{\mathrm{CM}}=(2.2 \pm 0.8) \times 10^{-19} \mathrm{~T}^{-2}$ at $3 \sigma$ confidence level for 34 refections inside the cavity, and $k_{\mathrm{CM}}=(3.2 \pm 1.3) \times 10^{-19} \mathrm{~T}^{-2}$ for 578 reflections. In 2008 a new measurement was published by the PVLAS collaboration using a Fabry-Pérot optical cavity and a superconducting magnet providing a $2.3 \mathrm{~T}$ field: $k_{\mathrm{CM}}=(1.4 \pm 2.4) \times 10^{-20} \mathrm{~T}^{-2}$ at $3 \sigma$ [प2]]. The same experiment at $5 \mathrm{~T}$ gave $k_{\mathrm{CM}}=(2.7 \pm 1.2) \times 10^{-20} \mathrm{~T}^{-2}$ at $3 \sigma$. More recently a new version 


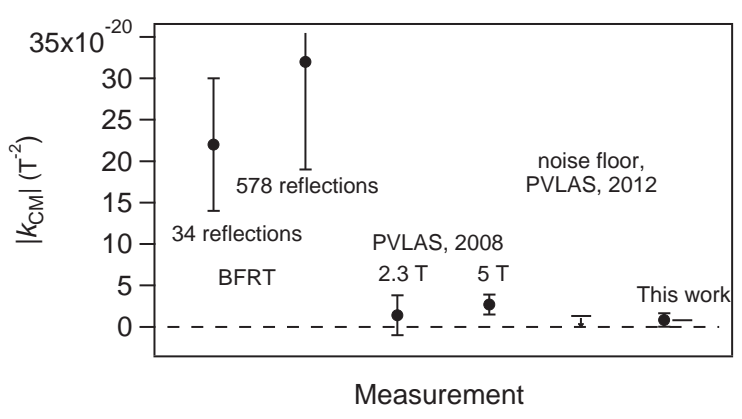

Figure 6: Comparison of reported absolute values of the vacuum magnetic linear birefringence and their uncertainties represented at $3 \sigma$. See text for details.

of the PVLAS apparatus based on two $2.5 \mathrm{~T}$ permanent magnets and a Fabry-Pérot optical cavity reached a noise floor corresponding to $k_{\mathrm{CM}}=1.3 \times 10^{-20} \mathrm{~T}^{-2}$ at $3 \sigma$, but "only when no spurious signal was observed" [ए]]. All these measurements are summarized in Fig. 6.

\section{Axions}

The study of photon propagation in transverse magnetic fields is also a powerful test of physics beyond the standard model. In particular, it has been predicted that photons in a magnetic field could oscillate via Primakoff effect into weakly interacting massive particles (WIMPs) like the axion. This hypothetical particle was introduced by Peccei and Quinn to solve the "strong CP problem" [[1]3] and it could be a possible constituent of dark matter.

Most stringent limits on axion or axion-like particles parameters, essentially its mass $m_{\mathrm{a}}$ and the coupling constant $g$ of axion-like particle to two photons, are given by astrophysical observations [44, [15]. But these limits depend on assumptions for the celestial sources. On the other hand, purely terrestrial experiments, where axions are produced and then detected on earth, are less sensitive but much more reliable since the experimental limits do not depend on any physical model.

Three kinds of purely terrestrial experiments exist. The first one corresponds to the "light shining through the wall" experiment [[6]. Up to now, the best limits have been obtained at DESY by the ALPS collaboration [[T], depicted as the grey line in Fig. $\square$. The area above the curve corresponds to the excluded region. The second kind of experiments consists in measuring the vacuum magnetic dichroism, i.e. the light absorption in vacuum depending on the light polarisation due to the presence of a transverse magnetic field. The most advanced experiment is performed by the PVLAS collaboration [ए2, प0]].

The third kind of experiment, complementary to the first ones, consists in measuring the vacuum magnetic birefringence, as described in this paper. Indeed, photon oscillations into a virtual massive particle like axions also induce an ellipticity signal in such an apparatus [एष]]. This ellipticity can be written as []]]:

$$
\Psi=\frac{2 F}{\pi} \frac{\Delta_{\mathrm{g}}^{2}}{2 \Delta_{\mathrm{osc}}} L_{B}\left(1-\frac{\sin \left[\Delta_{\mathrm{osc}} L_{B}\right]}{\Delta_{\mathrm{osc}} L_{B}}\right)
$$




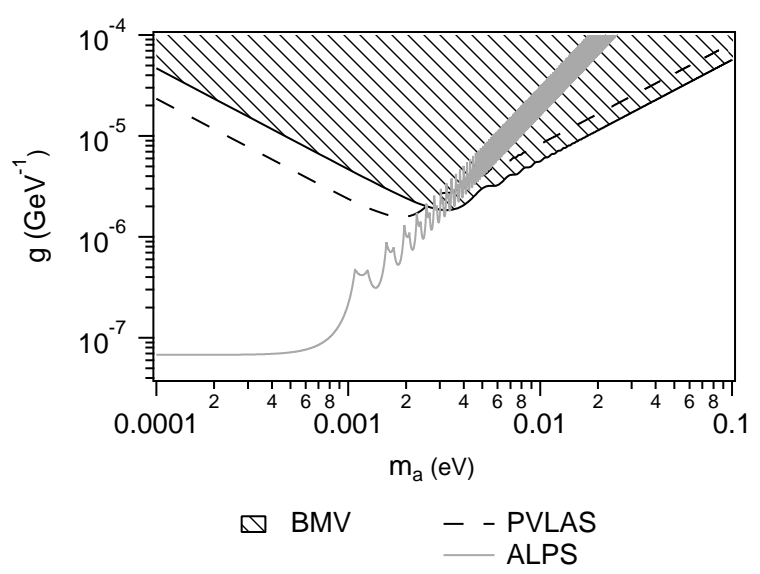

Figure 7: Limits at $3 \sigma$ confidence level on the axion-like particle-two photons coupling constant $g$ as a function of the particle mass $m_{\mathrm{a}}$ obtained by purely terrestrial experiments. Excluded regions are above the line. Solid black line and striped area: our limit; dashed line: limit given by the PVLAS collaboration; grey line: limit given by the APLS collaboration. See text for details.

with $\Delta_{g}=g B / 2, \Delta_{\text {osc }}=m_{\mathrm{a}}^{2} / 2 \omega$ and $\omega$ the photon energy. Our current limit obtained in vacuum and given in Eq. (B.6) corresponds to an ellipticity limit of $\Psi<1.5 \times 10^{-8} \mathrm{rad}$ at $3 \sigma$ confidence level. The black line and striped area in Fig. $\mathbb{D}$ corresponds to our current limit. The main advantage of this experiment is to extend limits for the heavier axion masses. The best limit is obtained at $m_{\mathrm{a}}=3.3 \mathrm{meV}$ with $g<1.9 \times 10^{-6} \mathrm{GeV}^{-1}$ at $3 \sigma$ confidence level.

\section{Conclusions and perspectives}

We presented the last advances of our BMV apparatus in terms of axion search, and the most precise measurement of vacuum magnetic birefringence ever realized. Our result validates our experimental method based on pulsed fields. In particular, it proves that the sensitivity obtained in a single pulse compensates the loss of duty cycle. To reach the QED value, the needed improvement is of three orders of magnitude. This is not conceivable with this first-generation experiment. Our strategy is therefore to increase the magnetic field thanks to the pulsed technology. At the moment, we have $B^{2} L_{B}=5.8 \mathrm{~T}^{2} \mathrm{~m}$ but we conceptualized and tested a pulsed coil prototype that has already reached a $B^{2} L_{B}$ higher than $300 \mathrm{~T}^{2} \mathrm{~m}$. Two coils of this type will be inserted in the experiment in the near future. This essential milestone really makes the vacuum birefringence measurement within our reach.

\section{Acknowledgments}

We thank all the members of the BMV collaboration, and in particular J. Béard, J. Billette, P. Frings, B. Griffe, J. Mauchain, M. Nardone, J.-P. Nicolin and G. Rikken for strong support. We are also indebted to the whole technical staff of LNCMI. We acknowledge the support of the Fondation pour la recherche IXCORE and the ANR-Programme non Thématique (Grant No. ANR-BLAN063-139634). 


\section{References}

[1] R. Battesti and C. Rizzo, Rep. Prog. Phys. 76, 016401 (2013).

[2] P. J. Mohr, B. N. Taylor, and D. B. Newell, Rev. Mod. Phys. 84, 1527 (2012).

[3] P. Berceau, M. Fouché, R. Battesti and C. Rizzo, Phys. Rev. A 85, 013837 (2012).

[4] R. Battesti, B. Pinto Da Souza, S. Batut, C. Robilliard, G. Bailly, C. Michel, M. Nardone, L. Pinard, O. Portugall, G. Trénec, J.-M. Mackowski, G. L. J. A. Rikken, J. Vigué and C. Rizzo, Eur. Phys. J. D 46, 323 (2008).

[5] A. Cadène, D. Sordes, P. Berceau, M. Fouché, R. Battesti and C. Rizzo, Phys. Rev. A 88, 043815 (2013).

[6] R. W. P. Drever, J. L. Hall, F. V. Kowalski, J. Hough, G. M. Ford, A. J. Munley and H. Ward, Appl. Phys. B 31, 97 (1983).

[7] S. Batut, J. Mauchain, R. Battesti, C. Robilliard, M. Fouché and O. Portugall, IEEE Trans. Applied Supercond. 18, 600 (2008).

[8] F. Bielsa, A. Dupays, M. Fouché, R. Battesti, C. Robilliard and C. Rizzo, Appl. Phys. B 97, 457 (2009).

[9] P. Berceau, M. Fouché, R. Battesti, F. Bielsa, J. Mauchain and C. Rizzo, Appl. Phys. B 100, 803 (2010).

[10] G. Zavattini, U. Gastaldi, R. Pengo, G. Ruoso, F. Della Valle and E. Milotti, Int. J. of Mod. Phys. A 27, 1260017 (2012).

[11] R. Cameron, G. Cantatore, A. C. Melissinos, G. Ruoso, Y. Semertzidis, H. J. Halama, D. M. Lazarus, A. G. Prodell, F. Nezrick, C. Rizzo and E. Zavattini, Phys. Rev. D 47, 3707 (1993).

[12] E. Zavattini, G. Zavattini, G. Ruoso, G. Raiteri, E. Polacco, E. Milotti, V. Lozza, M. Karuza, U. Gastaldi, G. Di Domenico, F. Della Valle, R. Cimino, S. Carusotto, G. Cantatore and M. Bregant, Phys. Rev. D 77, 032006 (2008).

[13] R. D. Peccei and H. R. Quinn, Phys. Rev. Lett. 38, 1440 (1977).

[14] The ADMX Collaboration: S. J. Asztalos, G. Carosi, C. Hagmann, D. Kinion, K. Van Bibber, M. Hotz, L. Rosenberg, G. Rybka, J. Hoskins, J. Hwang, P. Sikivie, D. B. Tanner, R. Bradley and J. Clarke, Phys. Rev. Lett. 104, 041301 (2010).

[15] E. Arik et al. (CAST Collaboration), Phys. Rev. Lett. 107, 261302 (2011).

[16] K. Van Bibber, N. R. Dagdeviren, S. E. Koonin, A. K. Kerman and H. N. Nelson, Phys. Rev. Lett. 59, 759 (1987).

[17] K. Ehret, M. Frede, S. Ghazaryan, M. Hildebrandt, E.-A. Knabbe, D. Kracht, A. Lindner, J. List, T. Meier, N. Meyer, D. Notz, J. Redondo, A. Ringwald, G. Wiedemann and B. Willke, Phys. Lett. B 689, 149 (2010).

[18] L. Maiani, R. Petronzio and E. Zavattini, Phys. Lett. B 175, 359 (1986). 